\title{
Obtenção e caracterização de copolímeros de Poliacrilonitrila (PAN) para uso em processos de fiação em extrusora
}

\author{
Obtaining and characterization of polyacrylonitrile \\ (PAN) copolymers for use in extruder \\ spinning processes
}

\author{
Robson Fleming ${ }^{1}$, Luiz Claudio Pardini ${ }^{2}$, \\ Nilton Granado ${ }^{3}$
}

\footnotetext{
${ }^{1}$ Instituto Federal de Mato Grosso do Sul - IFMS, Laboratório de Materiais e Metalurgia, CEP: 79310-110, Corumbá, Mato Grosso do Sul, Brasil.

${ }^{2}$ Departamento de Ciência e Tecnologia Aeroespacial, Instituto de Aeronáutica e Espaço, Instituto Tecnológico de Aeronáutica - DCTA/IAE/ITA, São José dos Campos, São Paulo, Brasil.

${ }^{3}$ Instituto Granado de Tecnologia da Poliacrilonitrila - IGTPAN, Jacareí, São Paulo, Brasil.

e-mail: robsonfleming@gmail.com, luizpardini@gmail.com, nilton@igtpan.com
}

\begin{abstract}
RESUMO
A disponibilidade de novos materiais, processos e equipamentos sempre traz perspectivas favoráveis ao mercado. A busca por processos alternativos e pela redução do custo da obtenção de precursores de materiais de alto desempenho tem se tornado primordial para o desenvolvimento do setor industrial de produtos com valores mais acessíveis, visando suprir a demanda de fibras de carbono de uso geral em um mercado que vem apresentando contínua expansão. As fibras de poliacrilonitrila (PAN) obtidas pelo processo de extrusão mostra potencial na redução de custo como precursor de fibras de carbono, em relação ao processo via fiação a úmido utilizado atualmente. Essa tecnologia inovadora permite a fiação por fusão da PAN empregando a glicerina (proveniente da produção do biodiesel) como seu principal plastificante, e dispensando o emprego de solventes altamente tóxicos. Assim sendo, o presente trabalho visou obter copolímeros de PAN que fossem adequados ao processo de fiação por extrusão convencional. Para tanto, foi utilizado para a obtenção dos copolímeros de PAN o processo de polimerização em suspensão aquosa e a caracterização foi realizada por meio da técnica de Cromatografia de Permeação em Gel (GPC). Os resultados mostraram que a técnica de GPC foi apropriada para determinar as propriedades moleculares dos copolímeros de PAN e que o comonômero estireno (ES) foi adequado para a polimerização, gerando copolímeros de PAN-co-ES com $\mathrm{M}_{\mathrm{n}}>40.000$ $\mathrm{g} / \mathrm{mol} \mathrm{e} \mathrm{M}_{\mathrm{w}}>130.000 \mathrm{~g} / \mathrm{mol}$.
\end{abstract}

Palavras-chave: Poliacrilonitrila, processos de polimerização, massa molar da PAN, fibras de PAN.

\begin{abstract}
The availability of new materials, processes and equipment always brings favorable perspectives to the market. The search for alternative processes and the precursor production cost reduction of high performance materials has become essential for the development of the industrial activity to produce the general purpose carbon fiber to supply the continuously expanding domestic market. The extrusion process of PAN fibers shows potential of cost reduction as it is a precursor of carbon fibers, in relation to the wet spinning method used up to now. This innovative technology enables spinning by melting PAN using glycerin (from biodiesel production) as the primary plasticizer, dispensing the use of highly toxic solvents. Thus, the present work aimed to obtain PAN copolymers that are suitable for the conventional extrusion spinning process. Therefore, PAN copolymer was copolymerized by aqueous suspension and the characterization was performed by Gel Permeation Chromatography (GPC) analysis. The results showed that GPC technique was suitable for determining of the PAN copolymers molecular properties and that styrene monomer was suitable for polymerization, generating PAN-co-ES copolymers with $\mathbf{M}_{\mathrm{n}}>40,000 \mathrm{~g} / \mathrm{mol}$ and $\mathbf{M}_{\mathrm{w}}>130,000 \mathrm{~g} / \mathrm{mol}$.
\end{abstract}

Keywords: Polyacrylonitrile, polymerization process, PAN molar mass, PAN fibers. 


\section{INTRODUÇÃO}

A poliacrilonitrila (PAN) foi sintetizada pela primeira vez em 1930 pelo Dr. Hans Fikentscher e o Dr. Claus Heuck em laboratórios de Ludwigshafen da empresa alemã IG Farben [1]. No entanto, como a PAN se mostrou insolúvel na maioria dos solventes comuns, a substância foi considerada inutilizável. Polímeros a base de PAN se caracterizam principalmente por não serem fusíveis, já que por aquecimento acima de $180^{\circ} \mathrm{C}$ ocorre a ciclização dos grupos nítrilicos $(\mathrm{CN})$ com grande liberação de energia e gases [2-5]. Dessa forma, sucessivas experiências têm tentado buscar uma solução para o problema da degradação da PAN antes de atingir sua temperatura de fusão.

Trabalhos anteriores de BRITO JÚNIOR et al. [5] e RIBEIRO et al. [6] demonstraram que o emprego de glicóis, como o 1,2,3-propanotriol (glicerol), atuam como um meio que retarda a ciclização iniciada no nitrogênio dos grupos nitrila. Além disso, substâncias altamente polares como a água, alcoóis e glicóis podem, hipoteticamente, atuar nas moléculas da PAN por meio da polaridade do hidrogênio que atrai, preferencialmente, o nitrogênio nitrílico interferindo na formação de ligações com o carbono vizinho e, consequentemente, na ciclização da cadeia. Entretanto, somente a presença do glicerol não garante um tempo suficiente para processos de termo conformação como a fiação de fibras por extrusão e moldagem por injeção. Portanto, é necessário a busca por copolímeros fusíveis de PAN e que poderiam ser processados em extrusoras disponíveis comercialmente.

Com relação à produção dos copolímeros de PAN, o processo é uma variação da polimerização em suspensão que é a técnica de polimerização amplamente utilizada para a produção de commodities, como o policloreto de vinila (PVC), poliestireno (PS), polimetacrilato de metila (PMMA) e a poliacrilonitrila (PAN) [8]. O processo mais utilizado na produção industrial do copolímero de PAN é conhecido como polimerização em suspensão aquosa ou dispersão aquosa. A principal diferença é que os iniciadores e dispersantes usados na polimerização por dispersão aquosa são solúveis em água, enquanto que na polimerização em suspensão são insolúveis em água [9-11]. O sistema redox mais comum utilizado na produção comercial de polímeros de PAN consiste de um oxidante (persulfato de amônia, ou de potássio, ou de sódio), de um agente redutor ou ativador (dióxido de enxofre, bissulfito de sódio ou metabissulfito de sódio) e de um catalisador (cátion ferroso) [9-11]. Segundo GUPTA [12] a utilização de pares redox como o bissulfito de sódio e persulfato de potássio tem se mostrado bastante efetivo nesta técnica, principalmente por serem exigidos em baixas concentrações e também apresentarem baixo custo. Normalmente nesses processos de síntese, utiliza-se um sistema redox que gera radicais livres num meio aquoso ácido na faixa de $\mathrm{pH}$ de 2 a 3,5, com temperatura relativamente baixa, na faixa de 40 a $60{ }^{\circ} \mathrm{C}[4,8,12]$.

Como os radicais livres reagem com o monômero gerando centros ativos primários, a concentração dos catalisadores afeta diretamente o crescimento da cadeia polimérica, influenciando nas propriedades físicas e mecânicas dos materiais em geral. Assim, o arranjo molecular e a configuração molecular são fatores determinantes nas propriedades dos polímeros [12-14]. Por exemplo, a massa molecular de um polímero é um fator preponderante nas propriedades do material a ser obtido. Polímeros acrílicos comerciais para aplicações de fibras têxteis apresentam massa molecular numérica média $\left(M_{n}\right)$ na faixa de 40.000 a 70.000 e massa molecular ponderal média $\left(M_{w}\right)$ entre 90.000 e 170.000 [8]. No entanto, quando se necessita da obtenção de fibras com maior resistência à tração e módulo de elasticidade como as fibras de carbono, as massas moleculares $M_{n}$ e $M_{w}$ apresentam valores acima de 100.000 e 250.000 , respectivamente. Dessa maneira, a estrutura dos polímeros tem sido objeto de muitos estudos e avaliações, sobretudo em relação ao tipo, tamanho e grau de organização da estrutura polimérica que é definida durante a sua polimerização e que pode influenciar nos processos posteriores de conformação.

Sendo assim, com a finalidade de buscar não somente aditivos para auxiliarem nos processos de fiação em extrusora, o presente trabalho tem como objetivo obter copolímeros de PAN (85\% Acrilonitrila/15\% Estireno) por meio de processos contínuo e por batelada e caracterizar os mesmos por meio de Cromatografia de Permeação em Gel (GPC).

\section{MATERIAIS E MÉTODOS}

\subsection{Materiais}

O copolímero de poliacrilonitrila (PAN) com estireno (ES) produzido por ambos os processos de polimerização está mostrado na Figura 1. A composição química do copolímero foi de $85 \%$ em massa do monômero acrilonitrila (AN) e $15 \%$ em massa do monômero estireno (ES). A acrilonitrila (AN) foi fornecida pela empresa Radicifibras (Brasil/SP), com pureza $>99,0 \%$ e o estireno foi fornecido pela empresa Quimlab Científica Ltda, com 99,9\% de pureza. 


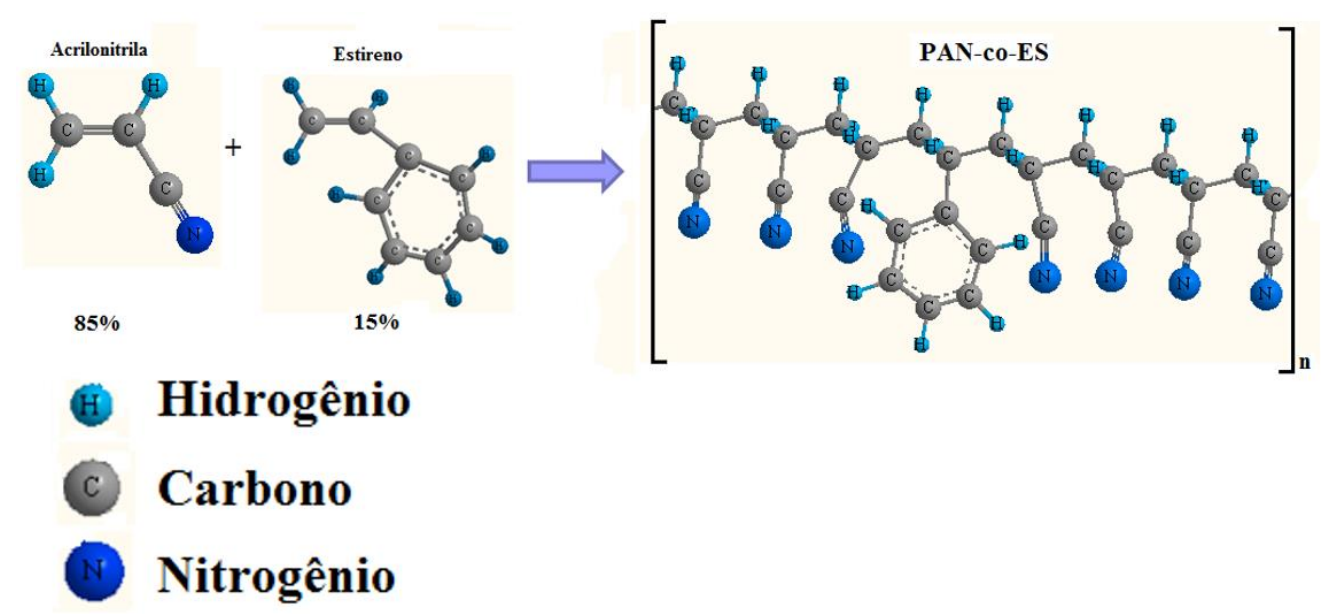

Figura 1: Provável estrutura do copolímero de PAN-co-ES.

\subsection{Polimerização em processo contínuo}

A Figura 2 mostra um esquema ilustrativo da instalação da planta piloto localizada na empresa Quimlab Científica. As partes principais do processo são um sistema de dosagem de reagentes (1), um reator de vaso de aço inoxidável de $27 \mathrm{~L}$, encamisado, com agitador eletromecânico de eixo vertical e fechado à pressão atmosférica (2), um sistema com coluna de esgotamento (3) e condensador (4) para monômeros não reagidos, reservatório para a coleta da solução polimérica (5) e reservatório para monômeros não reagidos (6).

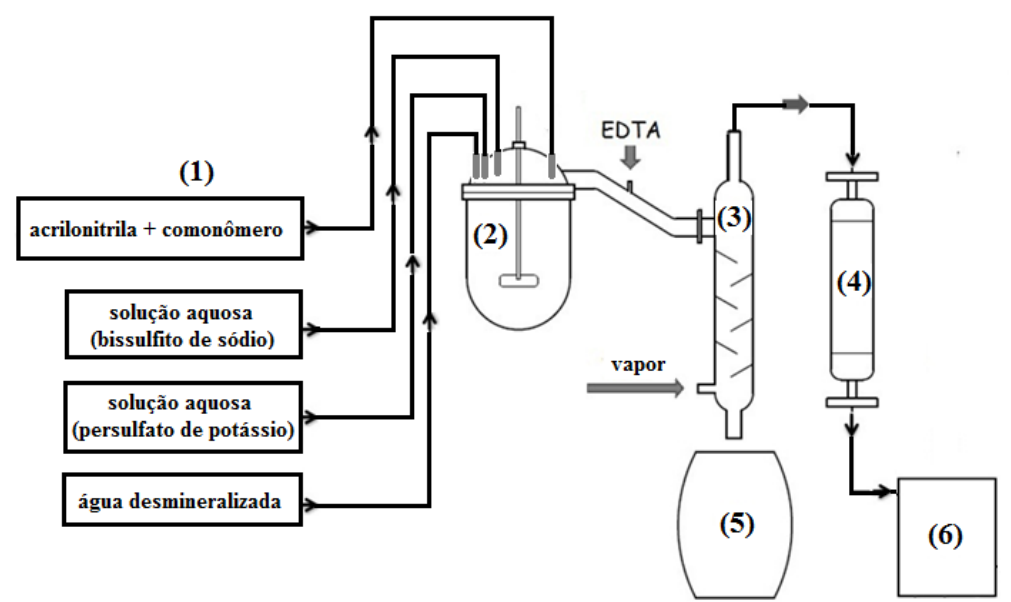

Figura 2: Esquema ilustrativo simplificado do processo de polimerização da PAN por meio do sistema redox.

A copolimerização por suspensão aquosa se iniciou preenchendo o reator com água desmineralizada e com os constituintes do sistema redox até $3 / 4$ do volume total do reator, pois a polimerização somente ocorre em fase aquosa. A solubilidade da acrilonitrila em água é de $8 \mathrm{~g} / 100 \mathrm{~mL}$ de água a $21^{\circ} \mathrm{C}$. A polimerização iniciou-se com um pH entre 2,7 e 3,0 e uma temperatura constante de $60{ }^{\circ} \mathrm{C}$. Diversos trabalhos informam a utilização da temperatura de $60{ }^{\circ} \mathrm{C}$ para a polimerização de copolímeros de PAN $[3,4,15]$.

Depois de alcançado as condições iniciais da síntese, os reagentes (acrilonitrila + comonômero), as soluções de bissulfito de sódio $(3,0 \mathrm{~g} / \mathrm{L})$ e de persulfato de potássio $(1,0 \mathrm{~g} / \mathrm{L})$ e a água desmineralizada foram introduzidos diretamente no fundo do reator químico por meio de bombas peristálticas. A reação de polimerização da PAN é altamente exotérmica e, portanto, o reator deve ser resfriado pela circulação de água proveniente de uma unidade de água gelada (chiller) que mantém, geralmente, a temperatura da reação constante entre 55 a $60{ }^{\circ} \mathrm{C}$. A viscosidade da suspensão de $\sim 50 \mathrm{mPa}$.s foi assegurada pela rotação do agitador entre 15 a $20 \mathrm{rpm}$. A polimerização continua até que o reator esteja complemente cheio de suspensão aquosa formada 
basicamente pelas partículas do polímero, água e monômeros que não reagiram e transborda por um duto tubular até a coluna de esgotamento ou coluna de destilação.

Neste duto foi transferida constantemente uma solução de ácido etilenodiaminotetraacético (EDTA) que age como agente complexante interrompendo a polimerização. A coluna de esgotamento tem função na reutilização dos monômeros não convertidos durante a polimerização. Assim, o fluxo ascendente de vapor superaquecido que entra na coluna de esgotamento promove a volatilização e o arraste dos comonômeros até um condensador. O condensador, previamente resfriado por circulação de água gelada, recuperou os comonômeros por condensação que foram coletados em um reservatório. A suspensão aquosa de polímero ao passar pela coluna de esgotamento foi coletada em um reservatório onde foi transferido para etapas posteriores (lavagem, filtração e secagem) que culminarão no polímero em pó seco de PAN.

\subsection{Polimerização por batelada}

O copolímero PAN-co-ES foi obtido pelo processo de polimerização via radicais livres (sistema redox), por meio do método de batelada em um vaporizador rotativo da marca Quimis, com balão de 1L, a uma temperatura constante de $60{ }^{\circ} \mathrm{C}$. A composição química inicial do copolímero foi a mesma do processo contínuo (85\% em massa do monômero acrilonitrila (AN) e $15 \%$ em massa do monômero estireno (ES)).

Inicialmente o balão de $1 \mathrm{~L}$ foi preenchido com $500 \mathrm{~mL}$ de água desmineralizada e aquecido até $60{ }^{\circ} \mathrm{C}$. Importante ressaltar que a polimerização somente ocorre em fase aquosa. Assim, com a temperatura constante, as soluções de bissulfito de sódio e de persulfato de potássio, nas concentrações desejadas, foram inseridas e o pH mantido entre 2,7 e 3,0. Por fim, foram inseridos os monômeros (AN+ES). A cada término de síntese foi utilizado EDTA para o encerramento das reações. Esses parâmetros foram utilizados para tentar manter uma similaridade com o processo contínuo utilizado na planta piloto.

A primeira etapa do estudo foi realizada fixando as soluções com uma concentração de 3,0 g/L para o bissulfito de sódio e 1,0 g/L para o persulfato de potássio, que foi a amostra controle denominada de PANco-ES-C. Os tempos para a síntese foram de $10 \mathrm{~min} ., 20 \mathrm{~min}$., $30 \mathrm{~min}$., $60 \mathrm{~min}$. e $120 \mathrm{~min}$. A segunda etapa foi realizada utilizando para o início da síntese as soluções contendo bissulfito de sódio e persulfato de potássio na proporção de 3/1 (amostra PAN-co-ES-C). A partir dessa proporção as amostras dos copolímeros foram obtidas variando a concentração das soluções em $20 \%$, conforme descrito na Tabela 1 . O tempo de síntese foi mantido constante em 30 min.

Tabela 1: Concentrações das soluções de bissulfito de sódio/persulfato de potássio utilizado na síntese por batelada.

\begin{tabular}{l|l|l}
\hline AMOSTRAS & $\begin{array}{l}\text { CONCENTRAÇÃO BISSULFITO } \\
\text { DE SÓDIO }(\mathbf{g} / \mathbf{L})\end{array}$ & $\begin{array}{l}\text { CONCENTRAÇÃO PERSULFATO } \\
\text { DE POTÁSSIO }(\mathbf{g} / \mathbf{L})\end{array}$ \\
\hline PAN-co-ES-A & 4,2 & 1,4 \\
\hline PAN-co-ES-B & 3,6 & 1,2 \\
\hline PAN-co-ES-C & 3,0 & 1,0 \\
\hline PAN-co-ES-D & 2,4 & 0,8 \\
\hline PAN-co-ES-E & 1,8 & 0,6 \\
\hline PAN-co-ES-F & 1,2 & 0,4 \\
\hline PAN-co-ES-G & 0,6 & 0,2 \\
\hline
\end{tabular}

\subsection{Cromatografia de Permeação em Gel (GPC)}

As análises de GPC das amostras do copolímero de PAN-co-ES foram realizadas em um equipamento Waters, com uma bomba isocrática modelo 600E e velocidade da fase móvel (DMF com LiBr) de $1 \mathrm{~mL} / \mathrm{min}$ com pressão de $2 \mathrm{kPa}$. As amostras foram injetadas por um injetor Rheodyne com loop de $100 \mu \mathrm{L}$. Foi utilizado um forno TCM/CHM da Waters, onde foram instaladas 1 pré-coluna $(50 \times 7,5 \mathrm{~mm})$ e duas colunas PLgel $(300 \times 7,5 \mathrm{~mm})$ em série com tamanho de partícula nominal de $10 \mu \mathrm{m}$ e poros do tipo MIXED-B (intervalo para $\mathrm{Mw}$ de 500 a $10.000 .000 \mathrm{~g} / \mathrm{mol}$ ). A temperatura para as colunas foi mantida a $60{ }^{\circ} \mathrm{C}$. Foi utilizado um refratômetro diferencial da HP (modelo HP 1047A), para medir as diferenças de índice de refração (IR) e um integrador da Viscotek OmniFace, responsável de converter os sinais analógicos de IR em sinais digitais por meio de um programa computacional OmniSEC que interpreta e calcula as informações coletadas do detector de IR. A calibração do equipamento foi realizada com sete padrões de óxido de polietileno (PEO), 
conforme mostra a Figura 3.

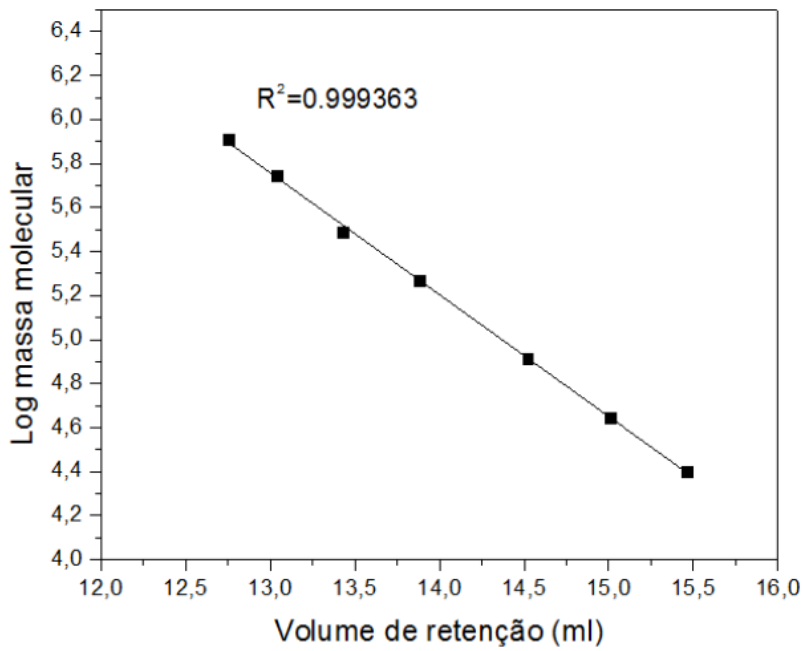

Figura 3: Curva de calibração para sete padrões PEO.

\subsection{Medida do rendimento (conversão) do copolímero obtido por batelada}

O procedimento para medir o rendimento do copolímero obtido por meio da polimerização por batelada está mostrado na Figura 4. Após cada síntese as soluções poliméricas foram colocadas sobre um conjunto de filtração. Este conjunto consistia basicamente de filtros de papel encaixados ao Kitassato de vidro presos por uma pinça de inox. Utilizou-se para a filtração uma bomba de vácuo Prismatec. O polímero foi lavado com água destilada para retirar os iniciadores e monômeros que não polimerizaram. Após esta etapa, o polímero foi inserido em uma estufa com temperatura em torno de $80{ }^{\circ} \mathrm{C}$ por $24 \mathrm{~h}$, para a retirada de umidade adsorvida. Assim, conseguiu-se medir o rendimento do polímero obtido medindo sua massa final em pó, conforme a equação (1).

$$
R(\%)=\frac{M_{P}}{M_{M}} \cdot 100
$$

Onde $M_{P}$ é a massa de polímero após síntese e secagem e $M_{M}$ é a massa de monômeros inseridos no início da síntese.

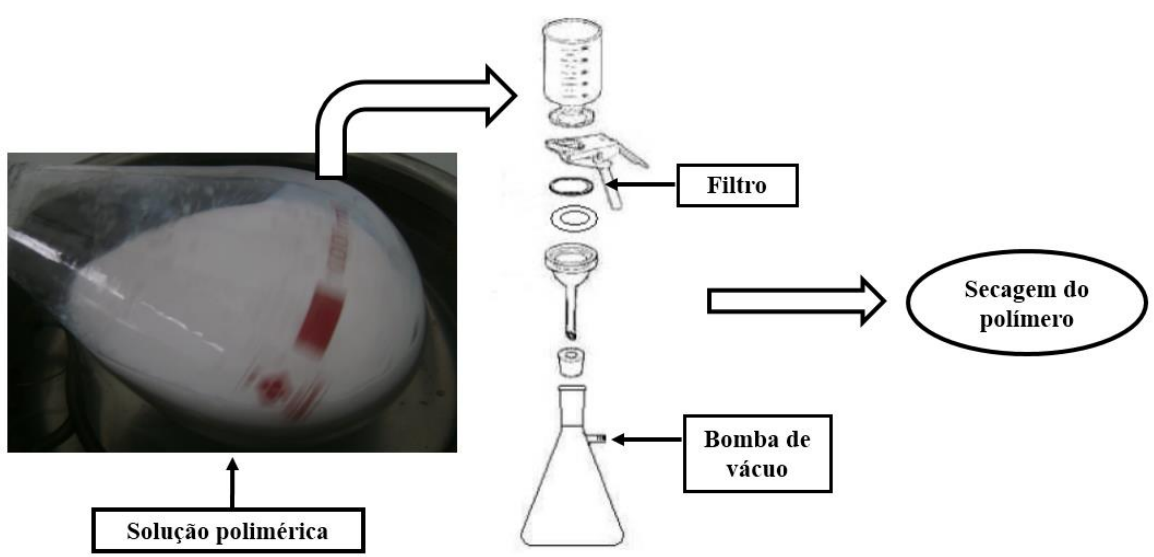

Figura 4: Procedimento utilizado para medir o rendimento (conversão) do copolímero. 


\section{RESULTADOS E DISCUSSÃO}

\subsection{Copolímeros de PAN-co-ES obtidos da planta piloto}

As curvas de GPC dos copolímeros de PAN-co-ES obtidos pelo sistema redox na planta piloto, com reator de 27 L, estão mostradas na Figura 5. Ressalta-se, que foram realizados 3 ciclos de $8 \mathrm{~h}$ de polimerização para tentar obter uma continuidade no processo. Amostras dos copolímeros de PAN-co-ES de cada ciclo foram retiradas e analisadas por GPC, para avaliar a distribuição da massa molar dos copolímeros obtidos. Os resultados da massa molar numérica média $\left(\mathrm{M}_{\mathrm{n}}\right)$, da massa molar ponderal média $\left(\mathrm{M}_{\mathrm{w}}\right)$ e do índice de polidispersividade $\left(\mathrm{M}_{\mathrm{w}} / \mathrm{M}_{n}\right)$ do copolímero PAN-co-ES estão mostrados na Tabela 2.

Verifica-se que as curvas GPC apresentam duas distribuições de massa molar, ou seja, os copolímeros obtidos apresentam tamanhos de cadeias distintos. A primeira faixa de massa molar compreendida entre 11,4 $\mathrm{mL}$ e 13,5 mL, com um pico em 12,6 mL, está associada à formação de copolímeros de alta massa molar, com $\mathrm{M}_{\mathrm{n}}$ acima de $720.000 \mathrm{~g} / \mathrm{mol}, \mathrm{M}_{\mathrm{w}}$ acima de $1.120 .000 \mathrm{~g} / \mathrm{mol}$ e um baixo índice de polidispersividade, em torno de 1,5. Por outro lado, a segunda distribuição de massa molar, com um pico em 15,1 mL, gerou uma quantidade maior de cadeias de copolímeros, mas de baixa massa molar, com $\mathrm{M}_{\mathrm{n}}$ e $\mathrm{M}_{\mathrm{w}}$ por volta de 15.000 $\mathrm{g} / \mathrm{mol}$ e $50.000 \mathrm{~g} / \mathrm{mol}$, respectivamente, e índices de polidispersividade mais elevados, por volta de 3,6.

Como a quantidade de copolímeros formados com baixa massa molar foi bem maior, estes copolímeros não apresentaram resultados satisfatórios no processo de extrusão. Esses copolímeros de PAN-co-ES geraram produtos com propriedades físicas insuficientes para a formação de pellets e consequentemente impossibilitando a obtenção de fibras.

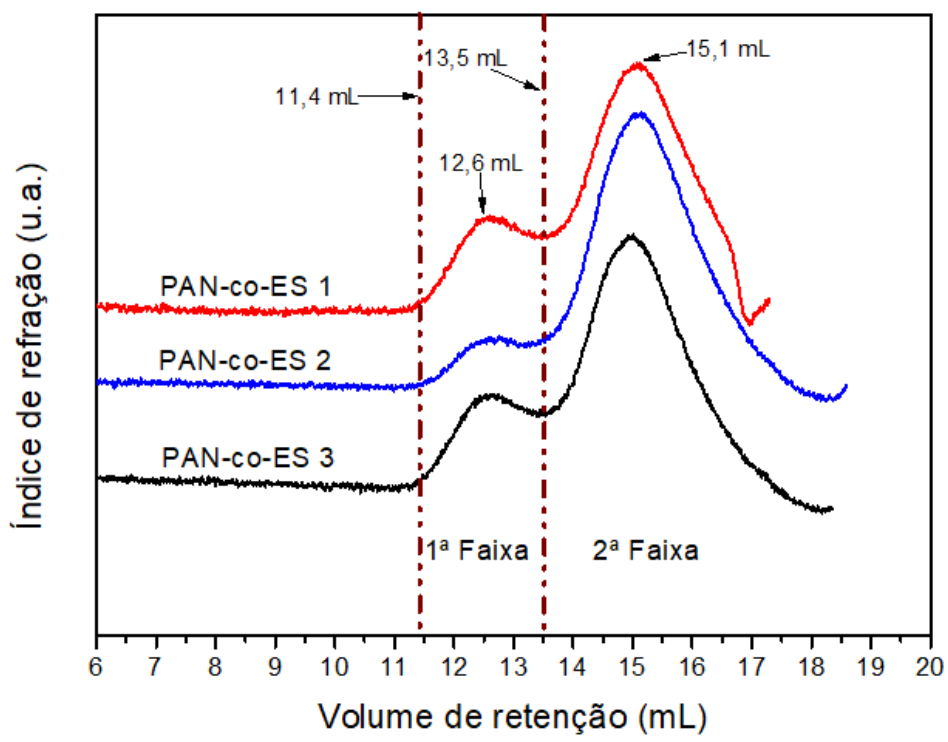

Figura 5: Curvas de distribuição da massa molar dos copolímeros de PAN-co-ES (planta piloto) obtidas por GPC.

Tabela 2: Resultados das massas molares das primeiras amostras de PAN-co-ES obtidas pela planta piloto.

\begin{tabular}{|c|c|c|c|c|c|c|}
\hline \multirow[b]{2}{*}{ AMOSTRAS } & \multicolumn{3}{|l|}{ 1 FAIXA } & \multicolumn{3}{|l|}{ 2a FAIXA } \\
\hline & $M_{n}(\mathrm{~g} / \mathrm{mol})$ & $M_{w}(g / m o l)$ & $M_{w} / M_{n}$ & $M_{n}(g / m o l)$ & $M_{w}(g / m o l)$ & $M_{w} / M_{n}$ \\
\hline PAN-co-ES 1 & 728.344 & 1.154 .000 & 1,584 & 21.575 & 57.964 & 2,687 \\
\hline PAN-co-ES 2 & 778.402 & 1.125 .000 & 1,445 & 12.152 & 52.550 & 4,324 \\
\hline PAN-co-ES 3 & 741.407 & 1.144 .000 & 1,543 & 14.827 & 59.469 & 4,011 \\
\hline Média & $749.087 \pm 25.965$ & $1.140 .936 \pm 14.731$ & $1,523 \pm 0,071$ & $15.724 \pm 4.856$ & $56.581 \pm 3.639$ & $3,599 \pm 0,869$ \\
\hline
\end{tabular}

A formação inadequada dos copolímeros PAN-co-ES 1, 2 e 3 impossibilitou sua utilização no processo de extrusão convencional. Entretanto, um novo ciclo de polimerização foi realizado com a mesma composição de monômeros ( 85 \% AN/15 \% ES) e efetuado nas mesmas condições de síntese, reduzindo somente a 
vazão das bombas dos iniciadores (bissulfito de sódio/persulfato de potássio), e assim reduzindo a sua concentração dentro do reator. O copolímero obtido também foi caracterizado por GPC e resultou em uma distribuição de massa molar de acordo com a Figura 6.

Observa-se que este copolímero, denominado de PAN-co-ES 4, apresenta expressiva redução de cadeias com maior massa molar, sobressaindo somente uma única distribuição de massa molar. Esse copolímero de PAN-co-ES apresenta valores de massa molar numérica média $\left(\mathrm{M}_{\mathrm{n}}\right)$ de aproximadamente $19.534 \mathrm{~g} / \mathrm{mol}$, massa molar ponderal média $\left(\mathrm{M}_{\mathrm{w}}\right)$ de $54.797 \mathrm{~g} / \mathrm{mol}$ e índice de polidispersividade de 2,805.

Esses valores de massa molar ainda são muito baixos para a produção de fibras acrílicas, principalmente para a produção de fibras acrílicas precursoras de fibras de carbono, pois irá gerar fibras de baixa resistência mecânica. De acordo com MASSON [8] e também GUPTA [11] copolímeros acrílicos comerciais para utilização em fibras têxteis apresentam massa molar numérica média $\left(\mathrm{M}_{\mathrm{n}}\right)$ na faixa de 40.000 a 70.000 $\mathrm{g} / \mathrm{mol}$ e massa molar ponderal média $\left(\mathrm{M}_{\mathrm{w}}\right)$ entre 90.000 e $170.000 \mathrm{~g} / \mathrm{mol}$. No entanto, quando se necessita da obtenção de fibras com maior resistência à tração e módulo como as fibras de carbono, as massas molares $\mathrm{M}_{\mathrm{n}}$ e $\mathrm{M}_{\mathrm{w}}$ apresentam valores acima de $100.000 \mathrm{~g} / \mathrm{mol}$ e $250.000 \mathrm{~g} / \mathrm{mol}$, respectivamente.

Assim sendo, apesar desse copolímero de PAN-co-ES apresentar boa homogeneidade com relação à distribuição de massa molar, quando utilizado no processo de extrusão, também resultou em propriedades físicas insuficientes para obtenção de pellets.

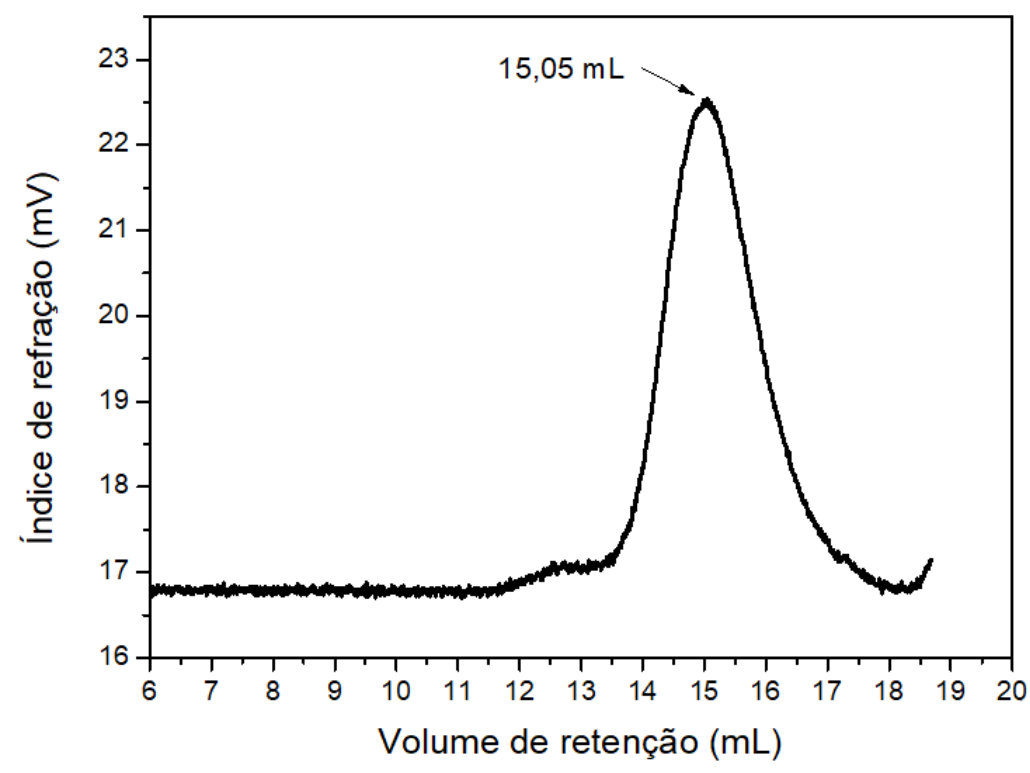

Figura 6: Curva de distribuição da massa molar do copolímero de PAN-co-ES 4 obtida por GPC (planta piloto com redução da vazão dos iniciadores).

\subsection{Copolímeros de PAN-co-ES obtidos por batelada}

A síntese de um polímero necessita de um controle rigoroso de diversas variáveis durante seu processo. As variáveis como temperatura, $\mathrm{pH}$ da solução, concentração de monômeros e iniciadores influenciam diretamente as propriedades dos polímeros a serem obtidos. A síntese em planta piloto só é adequada quando o processo está definido, controlado e estabilizado, porque gera alto custo durante a realização de ensaios, pois necessita de mão de obra qualificada (no mínimo três pessoas para operar a planta) e uma elevada quantidade de monômeros e iniciadores para a síntese.

Assim, devido às dificuldades em realizar ensaios em uma planta semi-industrial, uma solução encontrada foi reduzir as escalas dos ensaios para a escala laboratorial. Para tanto, o equipamento utilizado para as sínteses foi similar a um reator, mas com capacidade de 1 L. Como o processo foi realizado por batelada, com um rigoroso controle de temperatura, tempo e concentração dos iniciadores, foi possível obter maior variedade de polímeros, em menor tempo e menor custo.

Para avaliação das mudanças de massa molar do copolímero de PAN-co-ES, foram realizadas sínteses variando o tempo de permanência da solução polimérica no reator, e posteriormente sínteses com variação da 
concentração de iniciadores.

A Tabela 3 apresenta os resultados da síntese por batelada do copolímero de PAN-co-ES em diferentes tempos e concentração fixa de iniciadores em $3,0 \mathrm{~g} / \mathrm{L}$ para o bissulfito de sódio e $1,0 \mathrm{~g} / \mathrm{L}$ para o persulfato de potássio. A concentração na proporção 3/1 de bissulfito de sódio/persulfato de potássio era à concentração utilizada em polímeros comerciais na empresa Radici Fibras.

Observa-se que todas as massas molares $M_{n}$ apresentam valores em torno de $28.000 \mathrm{~g} / \mathrm{mol}$, não apresentando variação significativa. Porém, as massas molares $\mathrm{M}_{\mathrm{w}}$ apresentam aumentos crescentes e expressivos em relação ao aumento do tempo de síntese. A variação da massa molar $\mathbf{M}_{\mathrm{w}}$ entre os tempos de 10 min. e 120 min. de síntese é de aproximadamente $36.000 \mathrm{~g} / \mathrm{mol}$.

Um fator importante a ser verificado após a polimerização foi o rendimento (conversão) de polímero obtido. Essa conversão foi calculada de uma relação da quantidade de pó obtido pela quantidade de monômeros inseridos no início da síntese (equação (1)). A Figura 7 mostra os resultados do rendimento do copolímero de PAN-co-ES conforme aumenta o tempo de síntese. Verifica-se que o aumento do tempo de síntese de 10 min. para 120 min., contribui para que a quantidade de copolímero de PAN-co-ES obtido aumente de $54,8 \%$ para $86,3 \%$.

Tabela 3: Resultados da influência do tempo de síntese na massa molar do copolímero de PAN-co-ES obtido por batelada (concentração constante de bissulfito=3,0 $\mathrm{g} / \mathrm{L}$ e persulfato=1,0 $\mathrm{g} / \mathrm{L}$ ).

\begin{tabular}{l|l|l|l|l}
\hline AMOSTRAS & TEMPO $(\mathbf{m i n})$. & $\mathbf{M}_{\mathbf{n}}(\mathbf{g} / \mathbf{m o l})$ & $\mathbf{M}_{\mathbf{w}}(\mathbf{g} / \mathbf{m o l})$ & $\mathbf{M}_{\mathbf{w}} / \mathbf{M}_{\mathbf{n}}$ \\
\hline PAN-co-ES & 10 & 28.219 & 99.240 & 3,517 \\
\hline PAN-co-ES & 20 & 28.451 & 103.881 & 3,651 \\
\hline PAN-co-ES & 30 & 28.334 & 106.549 & 3,760 \\
\hline PAN-co-ES & 60 & 28.363 & 116.526 & 4,108 \\
\hline PAN-co-ES & 120 & 28.704 & 135.739 & 4,729 \\
\hline
\end{tabular}

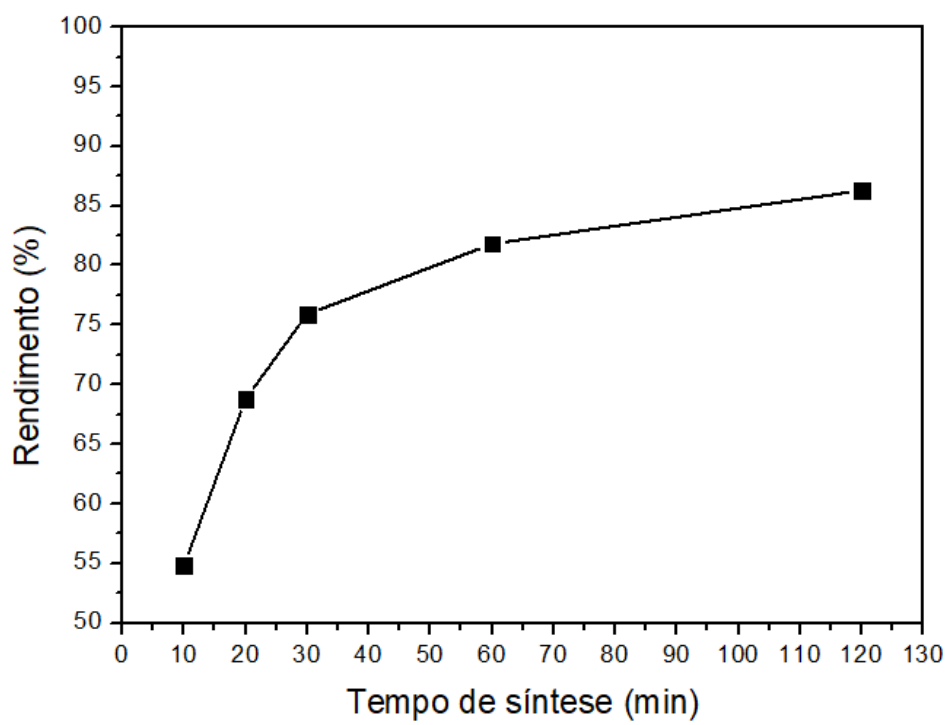

Figura 7: Influência do tempo de síntese no rendimento do copolímero de PAN-co-ES obtido por batelada (concentração constante de bissulfito $=3,0 \mathrm{~g} / \mathrm{L}$ e persulfato $=1,0 \mathrm{~g} / \mathrm{L}$ ).

Após a realização do estudo da influência no tempo de síntese, a próxima etapa foi verificar a influência da concentração dos iniciadores bissulfito de sódio/persulfato de potássio na síntese por batelada. As curvas de GPC dos copolímeros de PAN-co-ES obtidos pelo sistema redox em batelada estão mostradas na Figura 8. Verifica-se que as curvas apresentam uma adequada simetria, indicando uma apropriada preparação tanto das amostras quanto da estabilização do equipamento GPC. 


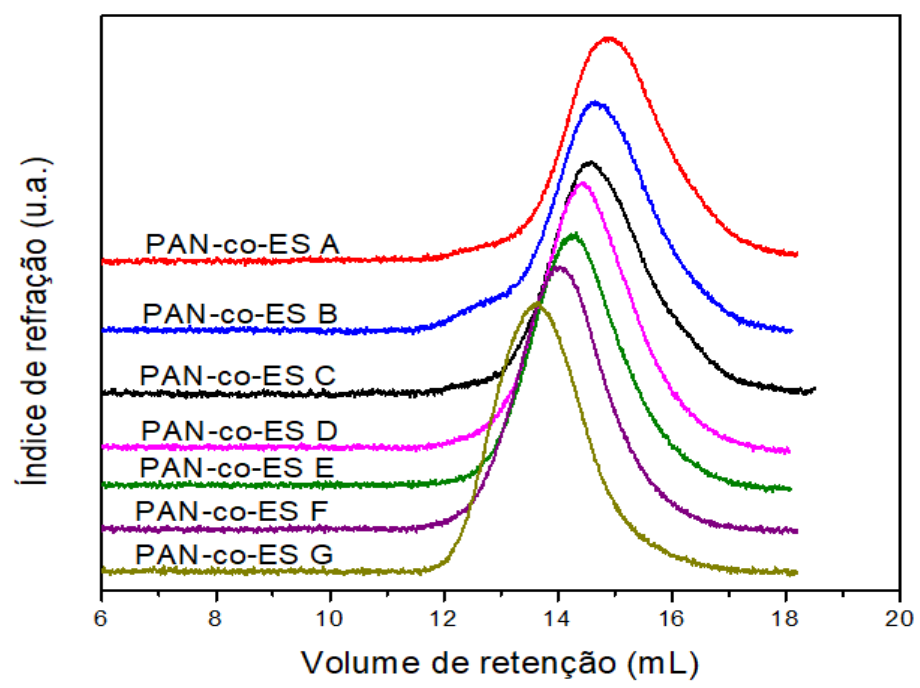

Figura 8: Curvas de distribuição da massa molar do copolímero de PAN-co-ES obtidas por GPC.

Os resultados obtidos a partir das curvas GPC são mostrados na Tabela 4 na forma de valores da massa molar numérica média $\left(\mathrm{M}_{\mathrm{n}}\right)$, da massa molar ponderal média $\left(\mathrm{M}_{\mathrm{w}}\right)$ e do índice de polidispersividade $\left(\mathrm{M}_{\mathrm{w}} / \mathrm{M}_{\mathrm{n}}\right)$ dos copolímeros de PAN-co-ES obtidos pelo processo de polimerização via sistema redox por batelada. Pode observar que reduzindo à concentração dos catalisadores do copolímero A (bissulfito de sódio $=4,2$ $\mathrm{g} / \mathrm{L}$ e persulfato de potássio $=1,4 \mathrm{~g} / \mathrm{L}$ ) para a concentração dos catalisadores do copolímero $\mathrm{G}$ (bissulfito de sódio=0,6 g/L e persulfato de potássio=0,2 g/L), a massa molar $\mathrm{M}_{\mathrm{n}}$ aumenta de $21.374 \mathrm{~g} / \mathrm{mol}$ para 95.370 $\mathrm{g} / \mathrm{mol}$, enquanto que a massa molar $\mathrm{M}_{\mathrm{w}}$ aumenta de $90.962 \mathrm{~g} / \mathrm{mol}$ para $329.040 \mathrm{~g} / \mathrm{mol}$. Outra observação interessante é a ocorrência de um aumento mais expressivo da massa molar do copolímero F para o copolímero $\mathrm{G}$ em relação ao aumento observado nos demais copolímeros.

Os resultados mostram que à medida que a concentração dos iniciadores da síntese é reduzida, maior é a massa molar do copolímero obtido. Isso é devido à maior concentração de iniciadores que corresponde a uma maior concentração de radicais livres, que disputam pela mesma quantidade de moléculas do monômero bloqueando a propagação da cadeia polimérica, já que a concentração do monômero é a mesma em todos os ensaios. Em consequência, há menor número de moléculas de acrilonitrila para cada radical, e, assim, menor é o tamanho das cadeias do copolímero obtido. De acordo com CHING [16], a ocorrência de cadeias mais curtas é devida à terminação prematura de propagação por efeito de radicais primários em excesso.

Outro efeito ocorre no índice de polidispersividade, que se reduz com a diminuição da concentração dos iniciadores. Observa-se que os copolímeros A e B apresentam o maior índice de polidispersividade, mostrando que isso pode ser um indicativo de uma excessiva geração de radicais livres durante a síntese desses copolímeros. Entretanto, os valores dos índices de polidispersividade de todos os copolímeros obtidos ainda estão dentro da faixa de distribuição de massa molar de polímeros de adição, que é aproximadamente entre 2 e 5 [17].

Verifica-se que a variação da concentração de bissulfito de sódio e persulfato de potássio além de influenciar a massa molar do copolímero, também influencia o rendimento (conversão) do copolímero de PANco-ES. Os copolímeros apresentam um rendimento entre 71 e $79 \%$, durante um tempo de síntese de 30 min., com exceção aos polímeros F e G, conforme pode ser observado pela Figura 9. As concentrações dos iniciadores dos copolímeros F e G geram rendimentos abaixo de $60 \%$ e $10 \%$, respectivamente. Dessa forma, embora a concentração do copolímero $\mathrm{G}$ resulte em massa molar com valores próximos a polímeros precursores de carbono $(\mathrm{Mn}>100.000 \mathrm{~g} / \mathrm{mol})$, seu rendimento é praticamente insignificante. 
Tabela 4: Resultados da análise de GPC dos copolímeros de PAN-co-ES, sintetizados por um tempo de 30 min., sob diferentes concentrações dos iniciadores.

\begin{tabular}{l|l|l|l|l|l}
\hline AMOSTRAS & $\begin{array}{l}\text { CONCENTRAÇÃO } \\
\text { BISSULFITO DE } \\
\text { SÓDIO (g/L) }\end{array}$ & $\begin{array}{l}\text { CONCENTRAÇÃO } \\
\text { PERSULFATO DE } \\
\text { POTÁSSIO }(\mathbf{g} / \mathbf{L})\end{array}$ & $\mathbf{M}_{\mathbf{n}}(\mathbf{g} / \mathbf{m o l})$ & $\mathbf{M}_{\mathbf{w}}(\mathbf{g} / \mathbf{m o l})$ & $\mathbf{M}_{\mathbf{w}} / \mathbf{M}_{\mathbf{n}}$ \\
\hline PAN-co-ES-A & 4,2 & 1,4 & 21.374 & 90.962 & 4,256 \\
\hline PAN-co-ES-B & 3,6 & 1,2 & 26.130 & 154.447 & 5,911 \\
\hline PAN-co-ES-C & 3,0 & 1,0 & 28.334 & 106.549 & 3,760 \\
\hline PAN-co-ES-D & 2,4 & 0,8 & 37.238 & 144.332 & 3,876 \\
\hline PAN-co-ES-E & 1,8 & 0,6 & 48.578 & 159.858 & 3,291 \\
\hline PAN-co-ES-F & 1,2 & 0,4 & 66.322 & 227.871 & 3,436 \\
\hline PAN-co-ES-G & 0,6 & 0,2 & 95.370 & 329.040 & 3,450 \\
\hline
\end{tabular}

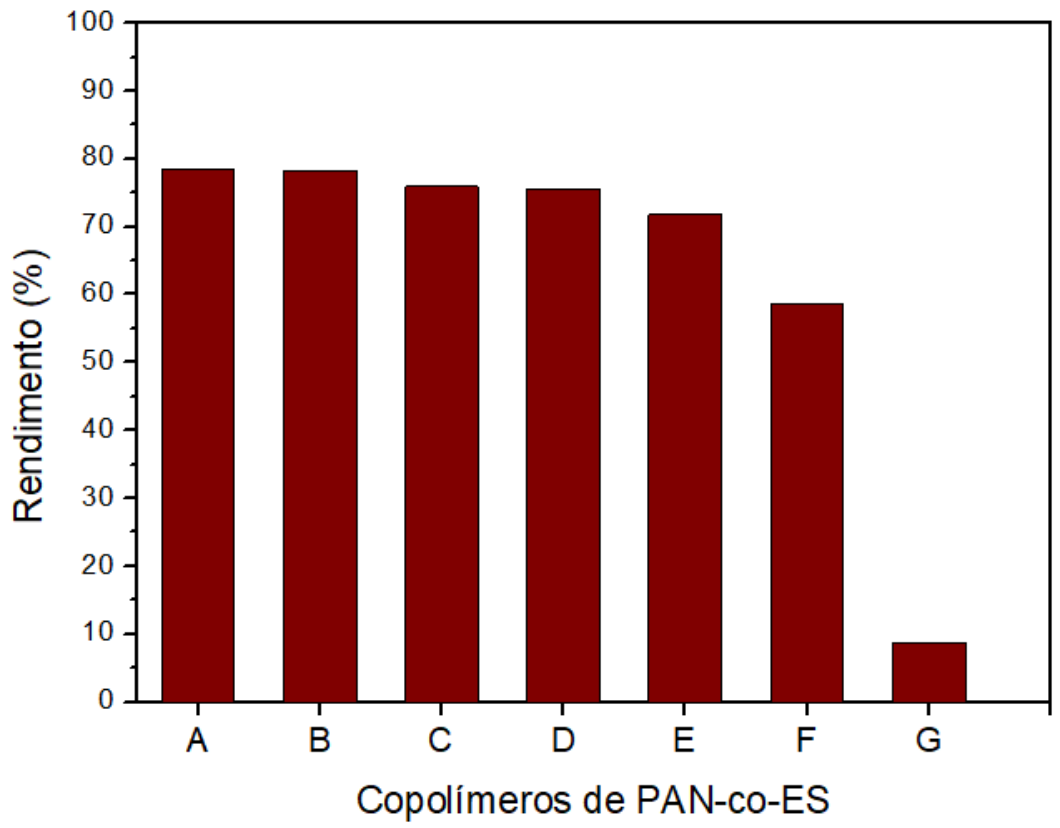

Figura 9: Rendimento dos copolímeros de PAN-co-ES obtidos por batelada, sintetizados por um tempo de 30 min., sob diferentes concentrações dos iniciadores.

\subsection{Obtenção e caracterização do copolímero de PAN-co-ES utilizado para a conformação de fibras em extrusora}

Após vários estudos com o copolímero de PAN-co-ES, foi definida uma concentração de iniciadores da síntese em 2,4 g/L de bissulfito de sódio e $0,8 \mathrm{~g} / \mathrm{L}$ de persulfato de potássio para ser utilizada na planta piloto. Essa concentração de iniciadores foi escolhida devido ao fato de se ter obtido um copolímero com massa molar $\mathrm{M}_{\mathrm{n}}$ de $\sim 37.000 \mathrm{~g} / \mathrm{mol}$ e $\mathrm{M}_{\mathrm{w}}$ de $\sim 144.000 \mathrm{~g} / \mathrm{mol}$ e um rendimento de $75 \%$ (amostra de PAN-co-ES D). Esse valor de massa molar foi próximo aos copolímeros de PAN utilizados para obtenção de fibras acrílicas.

Definida a concentração de iniciadores e mantendo as mesmas condições dos parâmetros do processo contínuo, foi realizado um único ciclo de $8 \mathrm{~h}$ de polimerização e retiradas amostras após 2 h do início do processo (amostra 1) e $30 \mathrm{~min}$. antes do término do processo (amostra 2).

A Figura 10 mostra a análise GPC dos copolímeros obtidos durante o ciclo de polimerização e a Tabela 5 apresenta seus respectivos valores. Observa-se que as curvas apresentam distribuição de massa molar bastante similares, com uma pequena diferença para a curva da amostra 1, que apresenta um pequeno ombro no início da curva gaussiana. O copolímero retirado para análise no início da polimerização (amostra 1) apre- 
senta valores da massa molar $\mathrm{M}_{\mathrm{n}}$ de $43.640 \mathrm{~g} / \mathrm{mol}$ e $\mathrm{M}_{\mathrm{w}}$ de $160.452 \mathrm{~g} / \mathrm{mol}$, com índice de polidispersividade de 3,677. A segunda análise no copolímero (amostra 2), após aproximadamente $5 \mathrm{~h}$ da primeira análise, apresenta valores da massa molar $\mathrm{M}_{\mathrm{n}}$ de $47.627 \mathrm{~g} / \mathrm{mol} \mathrm{e} \mathrm{M}_{\mathrm{w}}$ de $116.516 \mathrm{~g} / \mathrm{mol}$, com índice de polidispersividade de 2,446 .

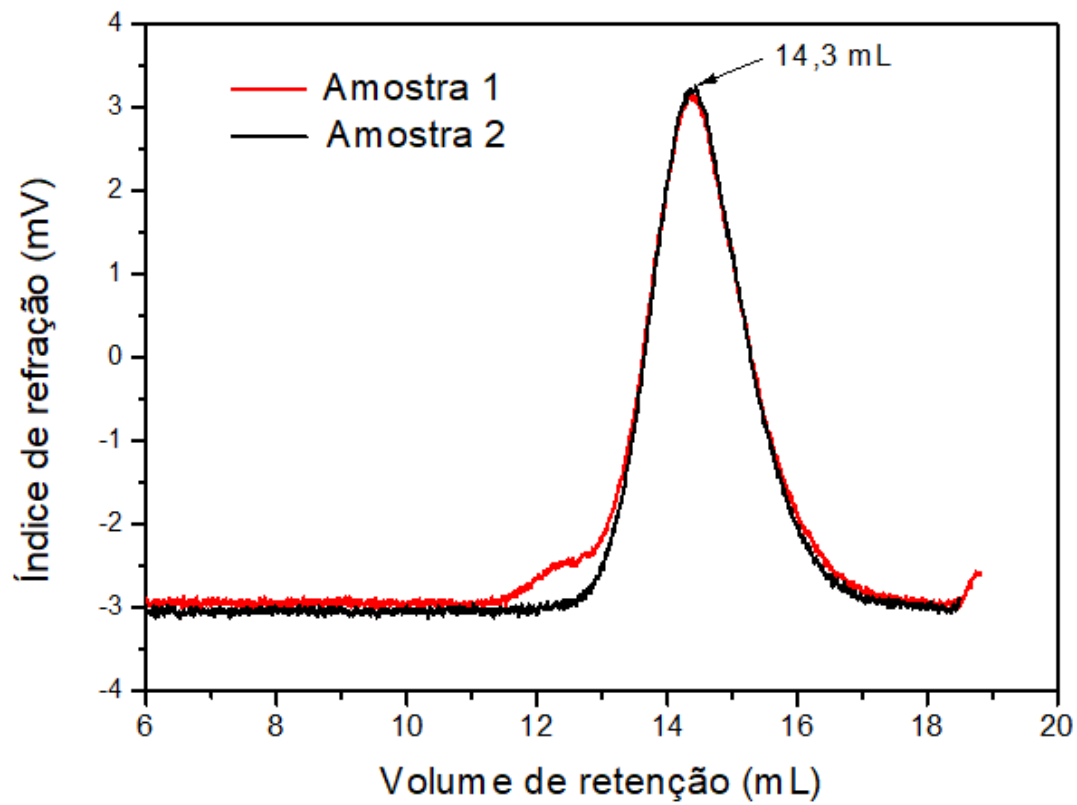

Figura 10: Curvas de distribuição da massa molar do copolímero de PAN-co-ES obtida por GPC (concentração de 2,4 $\mathrm{g} / \mathrm{L}$ bissulfito de sódio e $0,8 \mathrm{~g} / \mathrm{L}$ persulfato de potássio).

Tabela 5: Resultados da análise GPC dos copolímeros de PAN-co-ES obtidos pelo processo contínuo e que são adequados para obtenção de fibras pelo processo de extrusão.

\begin{tabular}{l|l|l|l}
\hline AMOSTRAS & $\mathbf{M}_{\mathbf{n}}(\mathbf{g} / \mathbf{m o l})$ & $\mathbf{M}_{\mathbf{w}}(\mathbf{g} / \mathbf{m o l})$ & $\mathbf{M}_{\mathbf{w}} / \mathbf{M}_{\mathbf{n}}$ \\
\hline Amostra 1 & 43.640 & 160.452 & 3,677 \\
\hline Amostra 2 & 47.627 & 116.516 & 2,446 \\
\hline Média & 45.634 & 138.484 & 3,034 \\
\hline
\end{tabular}

Esse processo de polimerização gerou copolímeros com massas molares similares aos copolímeros comerciais da empresa Radici Fibras $\left(\mathrm{M}_{\mathrm{n}} \sim 40.000 \mathrm{~g} / \mathrm{mol}\right)$. Ressalta-se que o foco do trabalho não foi à produção de fibras precursoras de carbono com relevante característica mecânica, visto que o precursor usado não foi o adequado para esta finalidade. Portanto, o estudo da polimerização do copolímero de PAN-co-ES teve como objetivo apenas obter um material que mantivesse integridade física e características mecânicas mínimas à fibra precursora produzida.

\section{CONCLUSÕES}

Copolímeros de acrilonitrila e estireno foram obtidos por meio de polimerização em suspensão aquosa, via sistema redox, em uma planta semi-piloto, com reator de $27 \mathrm{~L}$ e em escala laboratorial, com reator de $1 \mathrm{~L}$. Os resultados mostraram que reduzindo a concentração dos iniciadores bissulfito de sódio e persulfato de potássio na síntese, aumentou significativamente a massa molar do copolímero de PAN-co-ES. As massas molares $\mathrm{M}_{\mathrm{n}}$ aumentaram de $21.374 \mathrm{~g} / \mathrm{mol}$ para $95.370 \mathrm{~g} / \mathrm{mol}$, enquanto que as massas molares $\mathrm{M}_{\mathrm{w}}$ aumentaram de $90.962 \mathrm{~g} / \mathrm{mol}$ para $329.040 \mathrm{~g} / \mathrm{mol}$. Entretanto, a redução da concentração dos iniciadores reduziu o rendimento (conversão) do copolímero de maior massa molar para valores inferiores a $10 \%$.

As análises de GPC mostraram que copolímeros de PAN-co-ES com massas molares $\mathrm{M}_{\mathrm{n}}$ e $\mathrm{M}_{\mathrm{w}}$ por 
volta de $20.000 \mathrm{~g} / \mathrm{mol}$ e $60.000 \mathrm{~g} / \mathrm{mol}$, respectivamente, não foram adequadas para a obtenção de fibras de PAN pelo processo de extrusão convencional, pois resultaram em materiais com propriedades físicas inadequadas. As massas molares dos copolímeros de PAN-co-ES que tornaram possível à obtenção de fibras de PAN extrudadas de forma contínua e com boas propriedades físicas apresentaram valores de $M_{n}$ e $M_{w}$ de aproximadamente $46.000 \mathrm{~g} / \mathrm{mol}$ e $138.000 \mathrm{~g} / \mathrm{mol}$, respectivamente, com índice de polidispersividade em torno de 3,062 .

\section{AGRADECIMENTOS}

Agradecemos ao Instituto Granado de Tecnologia da Poliacrilonitrila pela viabilidade do presente trabalho e ao CNPq pelo suporte financeiro.

\section{BIBLIOGRAFIA}

[1] IGTPAN, INSTITUTO GRANADO DE TECNOLOGIA DA POLIACRILONITRILA, http://www.igtpan.com. Acessado em maio de 2018.

[2] MILLER, G. C., YU, J., JOSEPH, R. M., et al., "Melt-spinnable polyacrylonitrile copolymer precursors for carbon fibers", Polymer, v. 126, pp. 87-95, 2017.

[3] FU, Z., LIU, B., SUN, L., et al., "Study on the thermal oxidative stabilization reactions and the formed structures in polyacrylonitrile during thermal treatment", Polymer Degradation and Stability, v. 140, pp. 104$113,2017$.

[4] PARK, D. U., HAN, N. K., RYU, J. H., et al., "Spectroscopic analyses on chain structure and thermal stabilization behavior of acrylonitrile/methyl acrylate/itaconic acid-based copolymers synthesized by aqueous suspension polymerization", Fibers and Polymers, v. 18, n. 10, pp. 2007-2015, 2018.

[5] BRITO JÚNIOR, C. A. R., FLEMING, R. R., PARDINI, L. C., et al., "Poliacrilonitrila: processos de fiação empregados na indústria", Polímeros, v. 23, n. 6, pp. 764-770, 2013.

[6] RIBEIRO, R. F., PARDINI, L. C., ALVES, N. P., et al., "Thermal stabilization study of polyacrylonitrile fiber obtained by extrusion", Polimeros, v. 25, n. 6, pp. 523-530, 2015.

[7] CHAUDHARY, V, SHARMA, S., "Suspension polymerization technique: parameters affecting polymer properties and application in oxidation reactions", Journal of Polymer Research, v. 26, n. 102, 2019.

[8] MASSON, J. C., Acrylic fiber technology and applications, New York, Marcel Dekker, 1995.

[9] RWEI, S. P., WAY, T. F., CHING, W. Y., et al., "Effect of tacticity on the cyclization of polyacrylonitrile copolymers", Colloid Polym Sci, v. 295, pp. 803-815, 2017.

[10] PISIPATI, P., Synthesis and characterization of solution and melt processible poly (acrylonitrile-comethyl acrylate) statistical copolymers, PhD Thesis, Virginia Polytechnic Institute and State University, Blacksburg, VA, 2015.

[11] GUPTA, B. S., AFSHARI, M., "Polyacrylonitrile fibers", Handbook of Properties of Textile and Technical Fibres, 2 ed., chapter 15, North Carolina State University, Raleigh, NC, 2018.

[12] MORGAN, P.E., Carbon fibers and their composites, Taylor \& Francis Group, London, 2005.

[13] MORRIS, A., WEISENBERGER, M. C., ABDALLAH, M.G., et al., "High performance carbon fibers from very high molecular weight polyacrylonitrile precursors", Carbon, v.101, pp. 245-252, 2016.

[14] MUELLER, P. A., RICHARDS, J. R., CONGALIDIS, J. P., "Polymerization reactor modelling in industry", Macromolecular Reaction Engineering, v. 5, pp. 261-277, 2011.

[15] KOPEC, M., LAMSON, M., YUAN, R., et al., "Polyacrylonitrile-derived nanostructured carbon materials", Progress in Polymer Science, v. 92, pp. 89-134, 2019.

[16] CHING, L. S. Polimerização fotoquímica de acrilonitrila em suspensão aquosa, com o uso da radiação ultravioleta/visível, Dissertação de M.Sc., UNICAMP, Campinas, 1994.

[17] ODIAN, G., Principles of polymerization, 4 ed., John Wiley \& Sons, Inc., New Jersey, 2004. 


\section{ORCID}

Robson Fleming https://orcid.org/0000-0002-5257-6250

Luiz Claudio Pardini https://orcid.org/0000-0001-6982-1577

Nilton Granado

https://orcid.org/0000-0002-6662-2393 